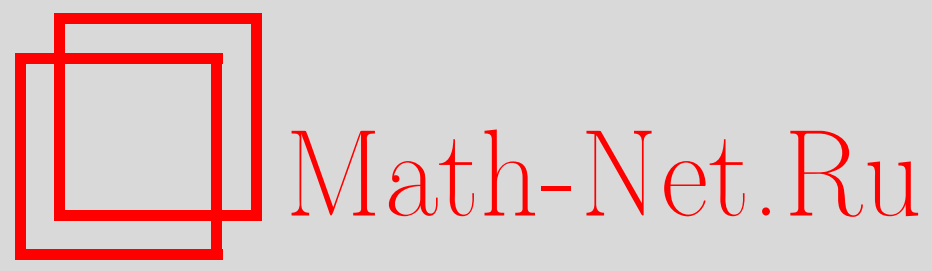

А. А. Грушо, Скрытые каналы и безопасность информации в компьютерных системах, Дискрет. матем., 1998, том 10, выпуск 1, 3-9

DOI: https://doi.org/10.4213/dm411

Использование Общероссийского математического портала Math-Net.Ru подразумевает, что вы прочитали и согласны с пользовательским соглашением http: //www .mathnet.ru/rus/agreement

Параметры загрузки:

IP: 3.80 .181 .102

26 апреля 2023 г., 12:51:43

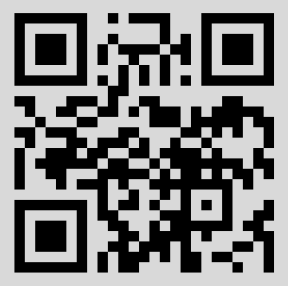




\title{
Скрытые каналы и безопасность информащии в компьютерных системах
}

\author{
(C) 1998 г. $\quad$ A. A. Грушо
}

\begin{abstract}
При построении систем защиты информационных технологий необходимо учитывать возможность наличия в таких системах так называемых троянских коней и других скрытых информационных воздействий. В работе моделируется такая ситуация при помощи пары автоматов, легального и нелегального, которые управляются общей входной последовательностью. Дается определение невидимости управления нелегального автомата. Изучаются условия существования и примеры невидимых каналов управления.

Работа выполнена при поддержке Российского фонда фундаментальных исследовании, грант 97-01-00162.
\end{abstract}

В основе построения гарантированной защиты информации в компьютерных системах лежит следующая идеология [1]. Для защиты от угроз безопасности информации в компьютерной системе формулируется набор правил обращения с этой информацией, выполнение которых не допускает реализации угроз. Эти правила называются политикой безопасности. Система защиты информации призвана поддерживать правила политики безопасности и считается хорошей, если она надежно поддерживает политику безопасности, и плохой в противном случае. В связи с тем, что основная часть компьютерных систем и программного обеспечения произведены за рубежом, в них могут содержаться скрытые программы (троянские кони), имеющие цель разрушить защиту. Поэтому можно предполагать, что использование импортного оборудования и программных средств в системах защиты не дает надежной поддержки политики безопасности. Чтобы исследовать возможности построения надежной поддержки политики безопасности из подобных ненадежных элементов, необходимо определить и проанализировать модели ненадежности. В работе исследуется один из подходов к данной задаче, который сводится к следующему. Ненадежность рассматривается как работа нелегального автомата $A 2$ (троянский конь, скрытое информационное воздействие, закладка и т.д.), функционирующего в легальной компьютерной системе $A 1$. Троянский конь в компьютерной системе общего назначения вряд ли создавался с ориентацией на конкретные приложения и вряд ли обладает большими интеллектуальными возможностями. Поэтому мы можем считать, что нелегальный автомат $A 2$, начиная действовать от команды извне или от логической бомбы, служит для того, чтобы через доступный канал связи осуществить прием, расположение в поле памяти и последующую передачу управления некоторому уже предметно-ориентированному программному 
обеспечению. Тогда через канал связи от внешнего злоумышленника к нелегальному автомату $A 2$ должно быть передано для $A 2$ значительное количество информации (нелегальные программы, вспомогательные данные и др.), но если такая передача будет замечена, то закладку легко уничтожить. Ясно, что передача информации автомату $A 2$ осуществляется через ту же входную последовательность, что и информация компьютерной системе $A 1$. За работой компьютерной системы $A 1$ наблюдает некоторый субъект $U$, который располагает некоторой функцией от текущего состояния автомате $A 1$ и поданного на вход задания.

Исследуются условия, когда $U$ не видит управления автоматом $A 2$ во входной последовательности автомата $A 1$, то есть когда канал управления автоматом $A 2$ является скрытым от $U$ (subliminal channel [2]).

Пусть дана компьютерная система $A 1$, которую можно представить конечным автоматом $(X, S, Y, \delta, \lambda)$, где $X$ - конечное множество входных сообщений, $S-$ множество состояний системы, $\delta: X \times S \rightarrow S$ - функция переходов, $y=\lambda(x, s)$, $x \in X, s \in S$, - функция выходов, которую может наблюдать субъект $U$, как след задания $x$ компьютерной системе $A 1$, находившейся в состоянии $s$. Мы будем говорить, что $U$ видит $\lambda(x, s), x \in X, s \in S$.

Нелегальный автомат $A 2=\left(Z, S_{1}, \delta_{1}\right)$ получает на вход такое же сообщение $x$ из $X$, как и автомат $A 1$. Однако на входе автомата $A 2$ стоит переводчик, преобразующий скрытый в $x$ сигнал управления в команду $z$ автомата $A 2$, то есть существует функция $g: X \rightarrow Z$, отображающая общий вход обоих автоматов во входной алфавит $Z$ автомата $A 2$. Будем считать $\lambda$ и $g$ сюръективными отображениями.

Предположим, что на $X$ задано распределение вероятностей $P_{X}(x)$, которое при помощи функции $\lambda(x, s)$ индуцирует распределения $Q_{s}(y)$ на $Y$ и $P_{Z}(z)$ на $Z$. Тем самым определены каналы от $X$ к $Y$ и $Z$, которые будем обозначать

$$
X \longmapsto Y, \quad X \longmapsto Z \text {. }
$$

Используя теоретико-информационные понятия, Шеннон [3] определил совершенный шифр. На наш взгляд, теоретико-информационные характеристики удачно подходят для математического описания секретов. Поэтому определим невидимость со стороны $U$ скрытого канала $X \longmapsto Z$ в терминах этих характеристик. Пусть $[3,4]$

$$
I_{s}(\mathrm{X}, Y)=H_{s}(Y)-H_{s}(Y \mid X)
$$

есть средняя взаимная информация схем $X$ и $Y$, где

$$
\begin{aligned}
H_{s}(Y) & =\sum_{y \in Y} Q_{s}(y) \log _{2} Q_{s}(y), \\
H_{s}(Y \mid X) & =\sum_{x \in X, y \in Y} P(x, y) \log _{2} \frac{P(x, y)}{P_{X}(x)} .
\end{aligned}
$$

Кроме того,

$$
I_{s}(X, Y \mid Z)=H_{s}(Y \mid Z)-H_{s}(Y \mid X, Z)
$$

- средняя взаимная информация схем $X$ и $Y$ при условии $Z$, где

$$
\begin{aligned}
H_{s}(Y \mid Z) & =\sum_{z \in Z, y \in Y} P(y, z) \log _{2} \frac{P(y, z x)}{Q_{s}(z)} \\
H_{s}(Y \mid X, Z) & =\sum_{x \in X, y \in Y, z \in Z} P(x, y, z) \log _{2} \frac{P(x, y, z)}{P(x, y)}
\end{aligned}
$$


$H_{s}(Y), H_{s}(Y \mid Z), H_{s}(X \mid X, Z)$ - энтропия и условные энтропии схемы $Y$ при условии $Z$ и $(Z, X), P$ без индекса означает, что распределение относится к нескольким схемам.

Определение 1 . Субъект $U$ не видит в состоянии $s$ скрытый канал $X \longmapsto Z$ тогда и только тогда, когда

$$
I_{s}(X, Y)=I_{s}(X, Y \mid Z) .
$$

Данное определение интерпретируется следующим образом. Если $A 2$ получает сообщение $z \in Z$ и не хочет, чтобы этот факт стал заметен субъекту $U$, то информационный поток от $X$ к $Y$ должен оставаться таким же, как в случае, когда канала $X \longmapsto Z$ просто нет. Положим

$$
\{y, z\}_{s}=\{x: \lambda(x, s)=y, g(x)=z\} .
$$

Лемма 1. Субвект $U$ не видит в состоянии $s$ скрытый канал $\mathrm{X} \longmapsto Z$ тогда $u$ только тогда, когда распределсние $P_{X}$ удовлетворяет условию: при всех у $\in Y$ и $z \in Z$

$$
P_{X}\left(\{y, z\}_{s}\right)=Q_{s}(y) P_{Z}(z)
$$

Доказательство. В силу функциональной зависимости $y$ и $z$ от $x$, для любого $s$

$$
H_{s}(Y \mid X, Z)=H_{s}(Y \mid X, g(X))=H_{s}(Y \mid X)=0 \text {. }
$$

Поэтому

$$
I_{s}(X, Y)=I_{s}(X, Y \mid Z) \Longleftrightarrow H_{s}(Y)=H_{s}(Y \mid Z)
$$

Равенство

$$
H_{s}(Y, Z)=H_{s}(Y)+H_{s}(Z)
$$

согласно [4] эквивалентно утверждению, что для каждого $(y, z)$ выполняется (1), что и требовалось доказать.

Когда $|\{y, z\}|=1$ для всех $y \in Y$ и $z \in Z$, в случае невидимого скрытого канала мера на $X$. задается соотношениями

$$
P_{X}(x)=Q_{s}(y) P_{Z}(z), \quad x \in X
$$

где

$$
y=\lambda(x, s), \quad z=g(x) .
$$

Значит, можно считать, что $X=Y \times Z$ и мера на $X$ определена формулой (2).

Теперь рассмотрим случай, когда начальное состояние $s$ автомата $A 1$ не фиксировано. Нас интересует вопрос о возможности построения канала, невидимого для $U$ при всех $s$. Для каждого $s \in S$ определена функция $y=\lambda(x, s)$ и определено понятие невидимости канала $X \longmapsto Z$ при начальном состоянии $s$. Как показывает следующий пример, одновременно для всех $s$ скрытого канала может не быть.

Пример 1. Пусть

$$
S=\left\{s_{1}, s_{2}\right\}, \quad X=\{0,1\}^{2}, \quad Y=\{0,1\}, \quad Z=\{0,1\}
$$




\section{Таблица 1.}

\begin{tabular}{cccc}
\hline$x$ & $\lambda\left(x, s_{1}\right)$ & $\lambda\left(x, s_{2}\right)$ & $g(x)$ \\
\hline 00 & 0 & 1 & 0 \\
01 & 0 & 1 & 1 \\
10 & 1 & 0 & 0 \\
11 & 1 & 0 & 1 \\
\hline
\end{tabular}

\section{Таблица 2.}

\begin{tabular}{cccc}
\hline$x$ & $\lambda\left(x, s_{1}\right)$ & $\lambda\left(x, s_{2}\right)$ & $g(x)$ \\
\hline 00 & 0 & 1 & 0 \\
01 & 1 & 1 & 1 \\
10 & 0 & 0 & 0 \\
11 & 1 & 0 & 1 \\
\hline
\end{tabular}

и на $X$ задана равновероятная мера. Таблицы 1 и 2 определяют все параметры двух вариантов систем $\{A 1, A 2\}$, из которых первая имеет для всех состояний невидимый скрытый канал, а для второй невидимый скрытый канал есть только для $\boldsymbol{s}_{2}$.

В случае, представленном таблицей 1 ,

$$
\begin{array}{ll}
H_{s_{1}}(Y)=I_{s_{1}}(X, Y)=1, & H_{s_{1}}(Y \mid Z)=I_{s_{1}}(X, Y \mid Z)=1, \\
H_{s_{2}}(Y)=I_{s_{2}}(X, Y)=1, & H_{s_{2}}(Y \mid Z)=I_{s_{2}}(X, Y \mid Z)=1,
\end{array}
$$

так что для состояний $s_{1}$ и $s_{2}$ канал $X \longmapsto Z$ невидим для $U$.

В случае, представленном таблицей 2 ,

$$
\begin{array}{ll}
H_{s_{1}}(Y)=I_{s_{1}}(X, Y)=1, & H_{s_{1}}(Y \mid Z)=I_{s_{1}}(X, Y \mid Z)=0, \\
H_{s_{2}}(Y)=I_{s_{2}}(X, Y)=1, & H_{s_{2}}(Y \mid Z)=I_{s_{2}}(X, Y \mid Z)=1,
\end{array}
$$

так что в состоянии $s_{2}$ канал $X \longmapsto Z$ невидим для $U$, а в состоянии $s_{1}$ канал $X \longmapsto Z$ видим для $U$.

Теорема 1. Пусть для любых $s \in S, y \in Y, z \in Z$

$$
\left|\{y, z\}_{s}\right|=1 \text {. }
$$

Схрытый канал $X \longmapsto Z$, невидимый для $U$ при всех $s \in S$, существует тогда $u$ только тогда, когда выполняются следующие условия.

(1) Cуществует $s_{0} \in S$, для которого существует скрытый канал $X \longmapsto Z$, невидимыน̆ для $U$.

(2) Для любого $s \neq s_{0}$ из $S$ совпадают множества значений вероятностей:

$$
\left\{Q_{s}(y)\right\}=\left\{Q_{s_{0}}(y)\right\}
$$


Доказательство. Чтобы доказать достаточность, возьмем произвольное $s \neq s_{0}$ и докажем, что в этом состоянии $U$ не видит канал $X \longmapsto Z$. Так как при $s_{0}$ существует канал $X \longmapsto Z$, невидимый для $U$, используя (3) и лемму 1 получим, что для любого $x \in X$

$$
P_{X}(x)=P_{X}\left(\left\{\lambda\left(x, s_{0}\right\},\left.g(x)\right|_{s_{0}}\right)=Q_{s_{0}}(y) P_{z}(z) .\right.
$$

Пусть $Y=\left\{y_{1}, \ldots, y_{n}\right\}$. Выберем произвольное $z \in Z$. Из (3) следует, что существуют различные элементы $x_{1}, \ldots, x_{n}$ из $X$ такие, что

$$
\begin{aligned}
x_{1} & =\left\{y_{1}, z\right\}_{s_{0}}, \\
& \ldots \\
x_{n} & =\left\{y_{n}, z\right\}_{s_{0}} .
\end{aligned}
$$

Эти соотношения определяют взаимно однозначное соответствие между множеством $g^{-1}(z)$ и $Y$. Для $s \neq s_{0}$

$$
\lambda\left(x_{1}, s\right)=y_{i_{1}}, \ldots, \lambda\left(x_{n}, s\right)=y_{i_{n}} .
$$

Опять из условия (3) следует, что в ряду (6) все образы различны, а это определяет еще одно взаимно однозначное соответствие между $Y$ и $g^{-1}(z)$. Используя (5), получаем, что для всех $j=1, \ldots, n$

$$
\mathbf{P}\left(\lambda\left(x_{j}, s\right)=y_{i_{j}} \mid g\left(x_{j}\right)=z\right)=\frac{P_{X}\left(x_{j}\right)}{P_{Z}(z)}=Q_{s_{0}}\left(y_{j}\right) .
$$

Из (4) следует, что $H_{s}(Y)=H_{s_{0}}(Y)$. Из (7) и (3) следует, что

$$
H_{s}(Y \mid Z)=\sum_{z \in Z} P_{Z}(z) H_{s}(Y)=H_{s}(Y)
$$

что доказывает достаточность.

Докажем необходимость. Очевидно, что первое условие выполняется. В силу леммы 1 и условия (3) для любых $s \in S$ и $x \in X$

$$
P_{X}(x)=Q_{s}(y) P_{Z}(z), \quad y=\lambda(x, s), \quad z=g(x) .
$$

Фиксируем $s_{0} \in S, z \in Z$ и будем для определенности считать, что

$$
g^{-1}(z)=\left\{x_{1}, \ldots, x_{n}\right\}
$$

так что $x_{i}=\left\{y_{i}, z\right\}_{s_{0}}$ при $i=1, \ldots, n$.

Тогда, используя взаимно однозначное соответствие $y_{i_{j}}=\lambda\left\{x_{j}, s\right\}, j=1, \ldots, n$, между $Y$ и $g^{-1}(z)$, получим, что

$$
P_{X}\left(x_{j}\right)=Q_{s}\left(y_{i_{j}}\right) P_{Z}(z)=Q_{s_{0}}\left(y_{j}\right) P_{Z}(z)
$$

Так как при $i=1, \ldots, n$ значения $\lambda\left(x_{i}, s\right)$ и $\lambda\left(x_{i}, s_{0}\right)$ пробегают все $Y$, из (9) получаем (4). Теорема доказана.

Рассмотрим два примера скрытых каналов, когда можно проверить и доказать их невидимость. 


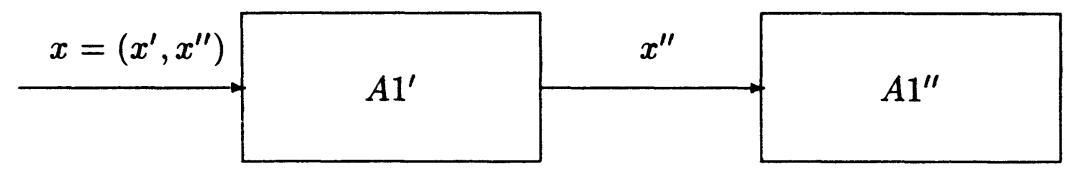

Рис. 1.

Пример 2. Пусть компьютерная система $A 1$ получает задание извне по сети связи и, следовательно, допускает иерархическую декомпозицию [1] по крайней мере на два или белее уровней (например, модель OSI или 4-хуровневая модель TCP/IP). Для таких систем верхний (прикладной) уровень не видит, что происходит на нижних уровнях, так как нижние уровни отвечают за организацию связи. Вместе с тем, вся информация верхних уровней проходит через подсистему нижнего уровня. Здесь отфильтровываются и отбрасываются данные, которые управляют связью. Поэтому моделью, связанной с сетью компьютерной системы $A 1$, можно считать представленное на рисунке последовательное соединение двух автоматов $A 1=\left(A 1^{\prime}, A 1^{\prime \prime}\right)$, где $A 1^{\prime \prime}$ - прикладной уровень, а $A 1^{\prime}$ отвечает совокупности уровней организации связи.

Для всех входных сообщений $x$ имеет место следующее представление в виде двух независимых частей: $x=\left(x^{\prime}, x^{\prime \prime}\right)$, где $x^{\prime}$ предназначено для организации связи, а $x^{\prime \prime}$ - это информация для прикладного процесса. Контрольный процесс $U$ находится на прикладном уровне и наблюдает функцию $y=\lambda^{\prime \prime}\left(x^{\prime \prime}, s^{\prime \prime}\right)$, где $s^{\prime \prime}-$ состояние автомата $A 1^{\prime \prime}, y$ - элемент выходного алфавита $Y$ автомата $A 1^{\prime \prime}, \lambda$ - это функция выхода автомата $A 1^{\prime \prime}$, зависящая от входного задания $x^{\prime \prime}$ и состояния $s^{\prime \prime}$ автомата $A 1^{\prime \prime}$. Другими словами, ьыходная функция $\lambda$ автомата $A 1$, находящегося в состоянии $s$, имеет виц

$$
y=\lambda\left(\left(x^{\prime}, x^{\prime \prime}\right), s\right)=\lambda^{\prime \prime}\left(x^{\prime \prime}, s^{\prime \prime}\right)
$$

Обмен сообщениями на прикладном и обслуживающем связь уровнях системы $A 1$ с другим абонентом сети можно считать независимым в статистическом смысле, то есть

$$
P\left(x^{\prime}, x^{\prime \prime}\right)=P\left(x^{\prime}\right) P\left(x^{\prime \prime}\right) .
$$

В этом случае, согласно лемме 1 , канал служебной информации $X \longmapsto X^{\prime}$ невидим на верхнем уровне для получателя $U$ сообщения $x^{\prime \prime}$ ири любом состоянии $s$ автомата $A 1$, то есть

$$
I_{s}\left(X, X^{\prime \prime}\right)=I_{s}\left(X, X^{\prime \prime} \mid X^{\prime}\right) .
$$

Поэтому для любого $s^{\prime \prime}$ наблюдатель $U$ не видит канал $X \longmapsto X^{\prime}$. Поэтому для нелегального автомата $A 2$, берущего информацию из потока сообщений на уровне организации связи, любой скрытый канал $X^{\prime} \longmapsto Z$, определяемый переводчиком $z=g\left(x^{\prime}\right)$, будет невидим для $U$ при любом $s^{\prime \prime}$, так как (см. [5])

$$
I_{s}\left(X, X^{\prime \prime}\right) \geqslant I_{s}\left(X, X^{\prime \prime} \mid g\left(X^{\prime}\right)\right) \geqslant I_{s}\left(X, X^{\prime \prime} \mid X^{\prime}\right) .
$$

Например, если в $X^{\prime}$ есть синонимы, выполняющие одну и ту же роль, но записываемые различно, то это различие может быть использовано переводчиком 
команд для управления нелггальным автоматом $A 2$, который распознает эти синонимы. Если бы $U$ находился на нижнем уровне, то увидеть этот скрытый канал было бы возможно, однахо на верхнем уровне этот канал невидим.

Пример 3. Пусть $A 1$ в ходе функционирования часто попадает в состояние $s_{0}$. Предположим, что противник, формируя задания для компьютерной системы $A 1$, знает, когда после выполнения очередного задания она должна перейти в состояние $s_{0}$. Будем считать, что при последовательности случайных заданий $X_{1}, X_{2}, \ldots$ среднее относительной частоты появления состояния $s_{0}$ равно $p>0$. Тогда наличия скрытого канала, не видимого для $U$ в состоянии $s_{0}$, достаточно, чтобы построить невидимый для $U$ скрытый канал управления автоматом $A 2$ с пропускной способностью $p H(Z)$. Если $A 1$ находится в состоянии $s_{0}$, то среднее количество информации, переданное по каналу $X \longmapsto Z$, равно $H(Z)$. Если состояние автомата $A 1$ есть $s \neq s_{0}$, то количество переданной информации равно нулю. В силу независимости подаваемых заданий по каналу $X \longmapsto Z$ количество информации от источника к получателю за $n$ заданий равно $t H(Z)$, где $t$ - число появлений состояния $s_{0}$. В среднем количество переданной информации равняется $n p H(Z)$. Значит, в среднем на одно сообщение приходится $p H(Z)$ бит информации для нелегального автомата $A 2$.

\section{Список литературы}

1. Грушо А. А., Тимонина Е. Е. Теоретические основъ защитъ информачии. Яхтсмен, Москва, 1996.

2. Schneier B. Applied Cryptography. Protocols, Algorithms and Source Code in C. Wiley, New York, 1996.

3. Шеннон К. Работы по теории ияформачии и кибернетике. ИЛ, Москва, 1963.

4. Вольфовиц Д. Теоремы кодирования теории информации. Мир, Москва, 1967.

Статья поступила 03.12.1997. 